\title{
Intracranial Calcification in an Elderly Woman with Carpopedal Spasm
}

\author{
James K. Young and Juan Maria Ibarra Co
}

Section of Endocrinology, Diabetes and Metabolism, Department of Internal Medicine, St. Luke's Medical Center, Philippines

A 66-year old woman presented to us with syncope and carpopedal spasm. The history of her condition started 10 years ago as recurrent loss of consciousness associated with carpopedal spasm occurring at 2-3 times in a year. A medical consult led to a diagnosis of hypocalcemia, and she has since been maintained on oral calcium supplementation. She was admitted to our center after her most recent syncopal attack which lasted for 20 minutes. At the emergency department, she had spontaneous recovery of consciousness without neurologic sequelae. Laboratory investigation showed normal complete blood count, serum sodium, potassium, creatinine and thyrotropin results. The assays for ionized calcium at 0.6 $\mathrm{mmol} / \mathrm{L}$ (nv -1.0-1.3) and serum magnesium at $1.7 \mathrm{mg} / \mathrm{dL}$ (nv 1.8-2.4) were low. Neither the electrocardiogram nor electroencephalogram showed any abnormality. Computed tomography scan of the brain demonstrated diffuse bilateral parenchymal calcification most prominent in both cerebro-cerebellar hemispheres and basal ganglia (Figure 1). She was given calcium gluconate intravenously and maintained on calcium and magnesium supplements and calcitriol per orem.

The patient is hypertensive and has a regular angiotensin receptor blocker. The patient underwent a subtotal thyroidectomy in 1975 for nodular goiter. She denies history of diabetes, cardiac, pulmonary, renal or gastrointestinal diseases. She also denies the same medical condition in the family.
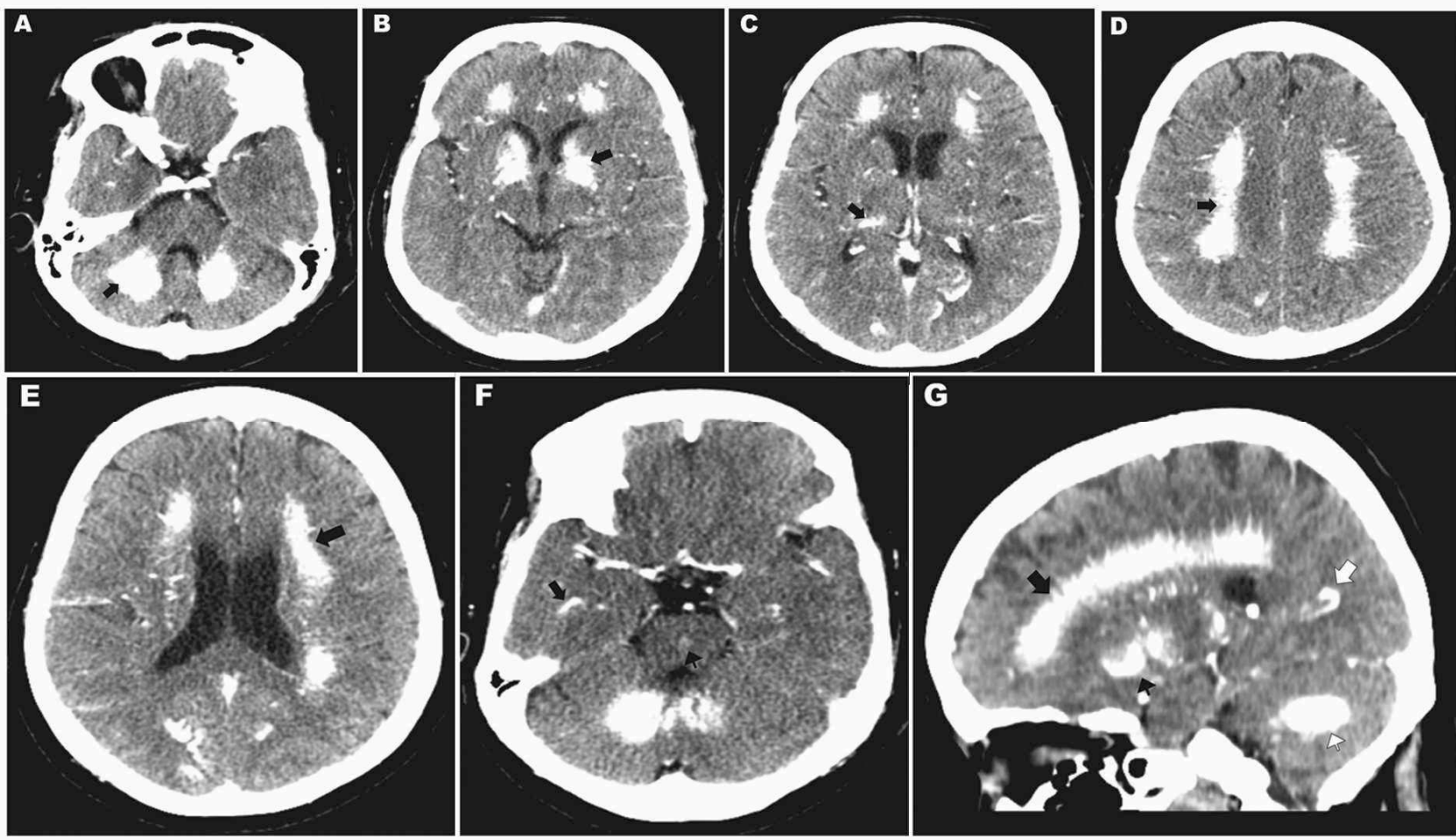

Figures 1A-F Axial view of a Cranial CT scan showing extensive calcification of both cerebellar hemispheres (arrow) in Panel A, bilateral capsuloganglionic regions (arrow) in Panel B, bilateral thalami (arrow) in Panel C, bilateral centrum semiovale (arrow) in Panel D, bilateral corona radiata (arrow) in Panel $E$ and at temporal (arrow) and pons (arrowhead) in Panel F. Figure $1 G$ is a sagittal view showing feathery calcification of centrum semiovale (black arrow), basal ganglia (black arrowhead), occipital (white arrow) and cerebellar regions.

ISSN 0857-1074

Copyright $\odot 2011$ by the JAFES

Received February 25, 2011. Accepted March 26, 2011.
Corresponding author: James K. Young, MD

Diabetes, Thyroid and Endocrine Center, $12^{\text {th }}$ Floor Cathedral Heights Building Complex, St. Luke's Medical Center, QC

Tel: 723-0101 loc 5210

Fax: 723-0101 loc 5210

Email: jameskyoung@doctor.com 
The clinical and imaging presentation of our case is compatible with the Fahr's syndrome. Fahr's syndrome refers to a rare neurodegenerative entity characterized as symmetric polytopic calcifications in one or more of the following areas: basal ganglia, cerebral white matter, thalami, internal capsulae, and cerebellum, which can lead to pyramidal, extrapyramidal, cerebellar symptoms, alteration of sensitive perception and psychiatric manifestations. ${ }^{1-6}$ There is no cure for Fahr's Syndrome, nor is there a standard course of treatment. ${ }^{7}$ Treatment addresses symptoms on an individual basis. 3,8

\section{References}

1. Manyam BV 2005. What is and what is not 'Fahr's disease'. Parkinsonism and Related Disorders 11:73-80

2. Lazar M, Ion DA, Streinu-Cercel A, Badarau AI 2009. Fahr's Syndrome: diagnosis issues in patients with unknown family history of disease. Rom J of Morph and Embry 50(3):425-428
3. Lam JSP, Fong SYY, Yiu GC, Wing YK 2007. Fahr's Disease: a differential diagnosis of frontal lobe syndrome. Hong Kong Med J 13:75-7

4. Kowdley KV, Coul BM, Orwall ES 1999. Cognitive impairment and intracranial calcification in chronic hypoparathyroidism. Am J Med Sci 317:273-277

5. Manyam BV, Bhatt MH, Moore WD, Devleschoward AB, Anderson DR, Calne DB 1992. Bilateral striopallidodentate calcinosis: cerebrospinal fluid, imaging, and electrophysiological studies. Ann Neurol 31:379-384.

6. Srivastava S, Bhatia MS, Sharma V, Mahajan S, Rajender G 2010. Fahr's Disease: An incidental finding in a case presenting with psychosis. German J Psychiatry 13 (2): 86-90)

7. Hasan A, Bajpai G, Varshney N 2007. Fahr's Disease. JIACM 8(3): 260-

8. Kono S, Manabe Y, Tanaka T, Fujii D, Sakai Y, Narai H, Omor N, Abe $\mathrm{K}$ 2009. A case of Fahr's disease presenting as chorea successfully treated by the use of quetiapine. Clinical Medicine: Case Reports 2:63-65

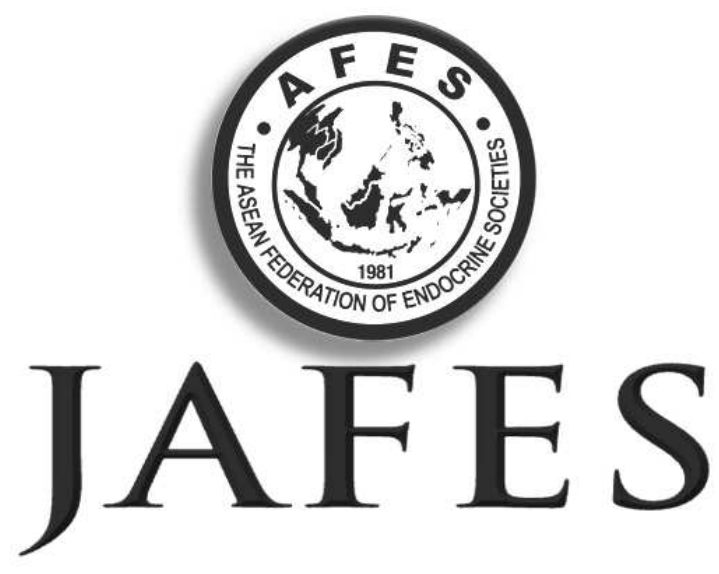

\section{Had an invigorating discussion in Grand Rounds? Share your Clinical Case Seminars at JAFES A Asia.com.}

